\title{
Interpolation and Sampling for Generalized Bergman Spaces on finite Riemann surfaces
}

Alexander Schuster and Dror Varolin

\begin{abstract}
We find sufficient conditions for a discrete sequence to be interpolating or sampling for certain generalized Bergman spaces on open Riemann surfaces. As in previous work of Bendtsson, Ortega- Cerdà, Seip, Wallsten and others, our conditions for interpolation and sampling are as follows: If a certain upper density of the sequence has value less that 1 , then the sequence is interpolating, while if a certain lower density has value greater than 1 , then the sequence is sampling.

Unlike previous works, we introduce a family of densities all of which provide sufficient conditions. Thus we obtain new results even in classical cases, some of which might be useful in industrial applications.

The main point of the article is to demonstrate the interaction between the potential theory of the Riemann surface and its interpolation and sampling properties.
\end{abstract}

\section{Introduction}

The goal of this paper is to establish sufficient conditions for a uniformly separated set on a finite Riemann surface to be interpolating or sampling for a generalized Bergman space of holomorphic functions on that surface.

Let us fix an open Riemann surface $X$. Much of the geometry used in the statements and proofs of our results arises from potential theory on $X$. If $X$ is hyperbolic, then $X$ admits a Green's function, while if $X$ is parabolic, then $X$ admits a so-called Evans Kernel. (See Section 2 for definitions.) After a

2000 Mathematics Subject Classification: 30F99, 30F15, 30F45.

Keywords: Finite Riemann surfaces, Green's function, Evans kernel, Beurling density, Ohsawa's theorem. 
normalization of the latter, both objects are unique, and we refer to either as the extremal fundamental solution $E(z, \zeta) .{ }^{1}$ Associated to this extremal fundamental solution is a distance $\rho(z, \zeta)=e^{E(z, \zeta)}$, and we denote by $D_{\varepsilon}(z)$ the $\varepsilon$-disk with respect to this distance. The geometry and potential theory we use in this paper is discussed in greater detail in Section 2.

To a conformal metric $g=e^{-\psi}|d z|^{2}$ on $X$, a smooth function $\varphi: X \rightarrow \mathbb{R}$ and a discrete subset $\Gamma \subset X$, uniformly separated with respect to the distance $\rho$ above, we associated the following two Hilbert spaces:

$$
\mathscr{B}_{X}^{2}=\mathscr{B}_{X}^{2}(\varphi, g):=\left\{h \in \mathcal{O}(X) ;\|h\|^{2}:=\int_{X}|h|^{2} e^{-\varphi} d A_{g}<+\infty\right\}
$$

and

$$
\mathfrak{B}_{\Gamma}^{2}=\mathfrak{B}_{\Gamma}^{2}(\varphi, g):=\left\{\left(s_{\gamma}\right)_{\gamma \in \Gamma} ; \sum_{\gamma \in \Gamma}\left|s_{\gamma}\right|^{2} e^{-\varphi(\gamma)} A_{g}\left(D_{\sigma}(\gamma)\right)<+\infty\right\},
$$

where $\mathcal{O}(X)$ denotes the set of holomorphic functions on $X$,

$$
A_{g}(S)=\int_{S} e^{-\psi} \frac{\sqrt{-1}}{2} d z \wedge d \bar{z} \quad \text { and } \quad \sigma=\sigma(\Gamma)=\frac{1}{4} \inf _{\gamma \neq \gamma^{\prime}} \rho\left(\gamma, \gamma^{\prime}\right)
$$

Definition 1.1. Let $X$ be an open Riemann surface with extremal fundamental solution $E$, let $\varphi$ be a weight function and $g$ a conformal metric, and let $\Gamma$ be a discrete set such that $\sigma(\Gamma)>0$.

1. We say that $\Gamma$ is an interpolation set if for every $\left(s_{\gamma}\right) \in \mathfrak{B}_{\Gamma}^{2}$ there exists $F \in \mathscr{B}_{X}^{2}$ such that for all $\gamma \in \Gamma, F(\gamma)=s_{\gamma}$.

2. We say that $\Gamma$ is a sampling set if there is a constant $M$ such that for all $F \in \mathscr{B}_{X}^{2}$,

$$
\frac{1}{M}\|F\|^{2} \leq \sum_{\gamma \in \Gamma}|F(\gamma)|^{2} e^{-\varphi(\gamma)} A_{g}\left(D_{\varepsilon}(\gamma)\right) \leq M\|F\|^{2}
$$

The potential theoretic data that $X$ comes equipped with gives rise to a special conformal metric $e^{\nu}|d z|^{2}$ which we call the fundamental metric (see Definition 2.3) and which plays a key role in our results.

Let

$$
R_{X}:=\left\{\begin{array}{cc}
1 & X \text { is hyperbolic } \\
+\infty & X \text { is parabolic }
\end{array}\right.
$$

\footnotetext{
${ }^{1}$ For the sake of brevity, we have been slightly inaccurate. In the parabolic case, we will end up selecting an Evans kernel for a partial compactification of our Riemann surface. See the remark in the first paragraph of Section 2 below.
} 
Definition 1.2. (1) For each locally integrable function $f:\left[0, R_{X}\right) \rightarrow[0, \infty)$ and each $r \in\left(0, R_{X}\right)$, let $c_{r}:=2 \pi \int_{0}^{r} t f(t) d t$ and define $\xi_{r}(z, \zeta)$ by

$$
\xi_{r}(z, \zeta) e^{-\nu} \sqrt{-1} d \zeta \wedge d \bar{\zeta}=\frac{1}{c_{r}} f\left(\rho_{z}(\zeta)\right) \mathbf{1}_{D_{r}(z)}(\zeta) d \rho_{z}(\zeta) \wedge * d \rho_{z}(\zeta),
$$

where $\mathbf{1}_{A}$ denotes the characteristic function of a set $A$.

(2) For a conformal metric $g=e^{-\psi}|d z|^{2}$ on $X$, we set

$$
\tau_{\psi}=e^{\nu-\psi} \text {. }
$$

(3) The relative curvature of the metric $g=e^{-\psi}|d z|^{2}$ is the $(1,1)$-form

$$
\Theta_{\psi}=\Delta \psi-\tau_{\psi}^{-1} \Delta \tau_{\psi}
$$

(4) A metric $g=e^{-\psi}|d z|^{2}$ on a Riemann surface is admissible if the function

$$
\mathfrak{D}_{\psi}^{2}:=\frac{e^{\psi}}{\tau_{\psi}}\left|\frac{\partial \tau_{\psi}}{\partial z}\right|^{2}
$$

is uniformly bounded on $X$.

(5) To every uniformly separated sequence (see section 4 for the definition) we associate the upper and lower densities

$$
D_{f}^{+}(\Gamma):=\limsup _{r \rightarrow R_{X}} \sup _{z \in X} \sum_{\gamma \in \Gamma} \frac{\frac{\pi}{2} \xi_{r}(\gamma, z)}{\left(e^{\nu}\left(\Delta \varphi+\Theta_{\psi}\right)\right)(z)},
$$

and

$$
D_{f}^{-}(\Gamma):=\liminf _{r \rightarrow R_{X}} \inf _{z \in X} \sum_{\gamma \in \Gamma} \frac{\frac{\pi}{2} \xi_{r}(\gamma, z)}{\left(e^{\nu}\left(\Delta \varphi+\Theta_{\psi}\right)\right)(z)} .
$$

Our main results can now be stated as follows.

Theorem 1. Let $X$ be a finite open Riemann surface with fundamental metric $e^{-\nu}|d z|^{2}$ and admissible metric $g=e^{-\psi}|d z|^{2}$. Let $\varphi$ be a weight function on $X$ such that, for some $c>1, \frac{1}{c} \leq e^{\nu} \Delta \varphi \leq c$ and $e^{\nu}\left(\Delta \varphi+\Theta_{\psi}\right) \geq \frac{1}{c}$. Then every uniformly separated sequence $\Gamma \subset X$ satisfying $D_{f}^{+}(\Gamma)<1$ is an interpolation sequence.

For the case of sampling, we must place an additional constraint on the metric $g$ beyond admissiblity, namely that $g$ is dominated by a multiple of the fundamental metric.

Theorem 2. Let $X$ be a finite open Riemann surface with fundamental metric $e^{-\nu}|d z|^{2}$ and an admissible metric $g=e^{-\psi}|d z|^{2}$ such that $e^{-\psi} \leq C e^{-\nu}$ for some constant $C>0$. Let $\varphi$ be a weight function on $X$ such that, for some $c>1, \frac{1}{c} \leq e^{\nu} \Delta \varphi \leq c$ and $e^{\nu}\left(\Delta \varphi+\Theta_{\psi}\right) \leq c$. Then every uniformly separated sequence $\Gamma \subset X$ satisfying $D_{f}^{-}(\Gamma)>1$ is a sampling sequence. 
Theorems 1 and 2 are generalizations, to the case of open finite Riemann surfaces, of the celebrated results of Seip and Seip-Wallstén [8, 9, 10], who proved these theorems for the Bargmann-Fock space, of entire holomorphic functions in $L^{2}\left(e^{-|z|^{2}}\right)$ and the Bergman space, of square integrable holomorphic functions on the unit disk. The theorems of Seip et al. were generalized by Berndtsson and Ortega-Cerda [1] to more general weights. Berndtsson and Ortega-Cerdà also gave different proofs, using $L^{2}$ methods. In this paper, we employ the $L^{2}$ approach as well. In more general contexts, Ohsawa has studied interpolation problems for $L^{2}$ sections of holomorphic vector bundles on Stein manifolds [4, 5], using $L^{2}$ methods.

The main point of the present paper is to demonstrate the key role that the potential theory and geometry of the fundamental metric of a Riemann surface play in the study of interpolation and sampling on that Riemann surface. The use of finite Riemann surfaces, as opposed to more general Riemann surfaces, is to establish certain bounds for the fundamental metric, and other related potential theoretic objects. We do not know whether these needed bounds hold in complete generality.

\section{Contents}

1. Introduction 499

2. Analytic geometry of Riemann surfaces 503

Extremal fundamental solutions $\quad 503$

The fundamental metric $\quad 504$

Green's Formula and mean values $\quad 504$

Ohsawa's $L^{2}$ estimates for $\bar{\partial} \quad 506$

3. Examples 508

The Euclidean plane $\quad 508$

The disk $\quad 509$

4. Finite Riemann surfaces 511

Definition and construction of finite Riemann surfaces 511

Analytic-geometric properties of finite Riemann surfaces 512

$\begin{array}{ll}\text { Discrete subsets in finite Riemann surfaces } & 517\end{array}$

5. Compact Riemann surfaces $\quad 518$

Cohomological criterion for interpolation and sampling $\quad 518$

Analytic proof of Proposition 5.1(1) 519

6. Functions and singular weights $\quad 519$

A local construction of a holomorphic function $\quad 519$

A function with poles along $\Gamma \quad 520$

A function with bumps along $\Gamma \quad 522$

7. Proof of Theorem $1 \quad 525$

8. Proof of Theorem $2 \quad 526$

References $\quad 529$ 


\section{Analytic geometry of Riemann surfaces}

\section{Extremal fundamental solutions}

We write

$$
\Delta:=\sqrt{-1} \partial \bar{\partial}
$$

for the Laplace operator (with complex analytic normalization). Let $\delta_{\zeta}$ denote the Dirac mass at $\zeta$. The following definition is standard.

Definition 2.1. The Green's function $G: X \times X \rightarrow[-\infty, 0)$ on a Riemann surface $X$ is the function with the following properties.

(a) For each $\zeta \in X, \Delta_{z} G(z, \zeta)=\frac{\pi}{2} \delta_{\zeta}(z)$.

(b) If $H: X \times X \rightarrow[-\infty, 0)$ is a function with property (a), then $H(z, w) \leq G(z, w)$ whenever $z \neq w$.

It is well-known that the Green's function is symmetric.

Recall that an open Riemann surface is said to be hyperbolic if it admits a bounded subharmonic function and parabolic otherwise. It is well known that a Riemann surface has a Green's function if and only if it is hyperbolic. Property (b) guarantees that the Green's function is unique.

Definition 2.2. An Evans kernel on a Riemann surface $X$ is a symmetric function $S: X \times X \rightarrow[-\infty,+\infty)$ with the following properties.

(a) For each $\zeta \in X, \Delta_{z} S(z, \zeta)=\frac{\pi}{2} \delta_{\zeta}(z)$.

(b) For each $r \in \mathbb{R}$ and $p \in X$, the level set $\{\zeta \in X ; S(\zeta, p)=r\}$ is compact and non-empty.

An open Riemann surface admits an Evans kernel if and only if it is parabolic (see [2] or [3, pp. 352]). Moreover, after prescribing (with somewhat limited possibility) the logarithmic singularity at infinity, the Evans kernel is unique up to an additive constant.

We shall use the notation $E: X \times X \rightarrow\left[-\infty, \log R_{X}\right)$ to denote either the Green's function or some chosen Evans kernel, depending on whether the Riemann surface is hyperbolic or parabolic, respectively.

Remark. In fact, this is not precisely what we will do. Below we will deal only with finite Riemann surfaces. If such a surface $X$ is parabolic, then it is a compact Riemann surface with a finite number of points removed. Thus there is a compact Riemann surface $\tilde{X}$ and points $\gamma_{1}, \ldots, \gamma_{N}$ such that $X=\tilde{X}-\left\{\gamma_{1}, \ldots, \gamma_{N}\right\}$. We will take the Evans kernel not for $X$, but for some $\tilde{X}-\gamma_{j}$ for some $j$. Thus if the surface has only one puncture, then we have no choice, but if the surface has multiple punctures, we choose one of these punctures to define our kernel. 
We shall refer to $E$ as the extremal fundamental solution for $X$.

Using the extremal fundamental solution, we define

$$
\rho_{z}(\zeta):=e^{E(z, \zeta)}, \quad D_{\varepsilon}(z):=\left\{\zeta \in X ; \rho_{z}(\zeta)<\varepsilon\right\} \quad \text { and } \quad S_{\varepsilon}(z)=\partial D_{\varepsilon}(z) .
$$

\section{The fundamental metric}

Definition 2.3. The fundamental metric $e^{-\nu}|d z|^{2}$ is given by the formula

$$
e^{-\nu(z)} d z \wedge d \bar{z}=\lim _{\zeta \rightarrow z} \partial \rho_{z}(\zeta) \wedge \bar{\partial} \rho_{z}(\zeta)
$$

Note that any solution of the equation

$$
\Delta_{z} E(z, \zeta)=\pi \delta_{z}(\zeta)
$$

locally has the form

$$
E(z, \zeta)=\log |z-\zeta|+h(z, \zeta)
$$

for some function $h(z, \zeta)$ that is Harmonic in $z$. If $E$ is symmetric, then so is $h$ and thus $h$ is also harmonic in $\zeta$.

Now,

$$
\begin{aligned}
\partial \rho_{z}(\zeta) & =\rho_{z}(\zeta)\left(\frac{-(\bar{z}-\bar{\zeta})}{2|z-\zeta|^{2}}+\frac{\partial h(z, \zeta)}{\partial \zeta}\right) d \zeta \\
& =e^{h(z, \zeta)}\left(\frac{-(\bar{z}-\bar{\zeta})}{2|z-\zeta|}+|z-\zeta| \frac{\partial h(z, \zeta)}{\partial \zeta}\right) d \zeta
\end{aligned}
$$

It follows that

$$
\nu(z)=-2 h(z, z)+2 \log 2
$$

\section{Green's Formula and mean values}

Recall that on a Riemann surface with a conformal metric $e^{-\psi}|d z|^{2}$, the Hodge star operator simplifies somewhat when expressed in analytic coordinates $z=x+\sqrt{-1} y$ : if $f$ is a real-valued function, $\alpha=\alpha_{1} d x+\alpha_{2} d y$ is a real 1-form and $\theta d x \wedge d y$ is a real 2-form, then one has

$$
\begin{aligned}
& * f=f d A_{g}=e^{-\psi} f d x \wedge d y \\
& *\left(\alpha_{1} d x+\alpha_{2} d y\right)=-\alpha_{2} d x+\alpha_{1} d y \\
& *(\theta d x \wedge d y)=e^{\psi} \theta
\end{aligned}
$$


Using these formulas, we have $2 \Delta=d * d$, and Green's formula can be written

$$
2 \int_{D} f \Delta h-h \Delta f=\int_{\partial D} f * d h-h * d f
$$

Let $X$ be an open Riemann surface and $Y \subset \subset X$ an open connected subset whose boundary consists of finitely many smooth Jordan curves. It is well known that the Green's function $G_{Y}$ for $Y$ exists and is continuous up to the boundary. Moreover, the exterior derivative $d\left(G_{Y}(\zeta, \cdot)\right)$ is also continuous up to the boundary.

Remark. One can construct the Green's function $G_{Y}$ from the extremal fundamental solution $E$ of $X$ as follows. Since $Y$ has smooth boundary, the Dirichlet Problem of harmonic extension from the boundary can be solved on $Y$. We then take $G_{Y}(\zeta, z):=E(\zeta, z)-h_{\zeta}(z)$, where $h_{\zeta}$ is the harmonic function in $Y$ that agrees with $E(\zeta, \cdot)$ on the boundary of $Y$.

We write $H_{r, \zeta}(z):=G_{D_{r}(\zeta)}(\zeta, z)$. In fact, the function $H_{r, \zeta}$ has a particularly simple form in terms of the extremal fundamental solution $E$ :

$$
H_{r, \zeta}(z)=E(z, \zeta)-\log r, \quad z \in D_{r}(\zeta) .
$$

Moreover, in this case we don't need to assume that $r$ is a regular value of $\rho_{\zeta}$.

Putting $D=D_{r}(z)$ and $h=H_{r, z}$ in (2.1) and using the definition of Green's function, we obtain the following lemma.

Lemma 2.4. Let $r<R_{X}$ and $\zeta \in X$. Then

$$
2 \pi f(z)=\int_{S_{r}(z)} f * d E_{z}+\int_{D_{r}(z)} H_{r, z} \Delta f
$$

In particular, if $f$ is subharmonic, then

$$
f(z) \leq \frac{1}{2 \pi} \int_{S_{r}(z)} f * d E_{z}
$$

with equality when $f$ is harmonic.

Let $\xi_{r}$ be as in Definition 1.2. Then for any function $F$, we have

$$
\begin{aligned}
\int_{X} F(w) \xi_{r}(z, w) e^{-\nu(w)} \sqrt{-1} d w \wedge d \bar{w} & =\frac{1}{c_{r}} \int_{D_{r}(z)} F f\left(\rho_{z}\right) d \rho_{z} \wedge * d \rho_{z} \\
& =\frac{1}{c_{r}} \int_{0}^{r} t f(t)\left(\int_{S_{t}(z)} F * d E_{z}\right) d t .
\end{aligned}
$$

Thus, in view of (2.4) of Lemma 2.4, we have the following Lemma. 
Lemma 2.5. If $h$ is subharmonic then

$$
h(z) \leq \int_{X} \xi_{r}(z, w) h(w) e^{-\nu(w)} \sqrt{-1} d w \wedge d \bar{w}
$$

with equality if $h$ is harmonic.

\section{Ohsawa's $L^{2}$ estimates for $\bar{\partial}$}

In our proof of the interpolation theorem, we require a theorem for solving $\bar{\partial}$ with certain $L^{2}$ estimates. The theorem we need is slightly sharper than Hörmander's Theorem, due to Ohsawa.

Theorem 2.6 (Ohsawa). Let $X$ be a Riemann surface with conformal metric $e^{-\psi}|d z|^{2}, V \rightarrow X$ a holomorphic line bundle with smooth Hermitian metric $e^{-\varphi}$, and $\tau$ a positive function. Suppose that for some $\varepsilon, \delta>0$,

$$
e^{\psi}\left(\tau \Delta(\varphi+\psi)-\Delta \tau-\varepsilon \frac{|\partial \tau|^{2}}{\tau}\right) \geq \delta .
$$

Then there exists a constant $C=C_{\delta, \varepsilon}$ such that for any $V$-valued $(0,1)$-form $\alpha$ satisfying

$$
\int_{X} e^{-\varphi} \sqrt{-1} \bar{\alpha} \wedge \alpha<+\infty
$$

the equation $\bar{\partial} U=\alpha$ has a solution satisfying

$$
\int_{X}|U|^{2} \frac{e^{-\varphi}}{\tau} d A_{g} \leq C \int_{X} e^{-\varphi} \sqrt{-1} \bar{\alpha} \wedge \alpha .
$$

In the case of Riemann surfaces there is a short proof of Ohsawa's theorem. Since a short proof is not easily accessible in the literature, we shall give one here.

Proof of Ohsawa's Theorem. Let us first endow $V \rightarrow X$ with the Hermitian metric $e^{-\xi}=\tau e^{-\varphi}$. With

$$
\bar{\nabla}(h d \bar{z}):=\left(h_{\bar{z}}+\psi_{\bar{z}} h\right) d \bar{z}^{\otimes 2}
$$

denoting the covariant $\bar{\partial}$ derivative, a straightforward calculation shows that the formal adjoints $\bar{\partial}^{*}$ of $\bar{\partial}$ and $\bar{\nabla}^{*}$ of $\bar{\nabla}$ are given by

$$
\bar{\partial}^{*}(h d \bar{z})=-e^{\psi}\left(\frac{\partial h}{\partial z}-\frac{\partial \xi}{\partial z} h\right) \text { and } \bar{\nabla}^{*}\left(h d \bar{z}^{\otimes 2}\right)=-e^{\psi}\left(\frac{\partial h}{\partial z}-\frac{\partial \xi}{\partial z} h\right) d \bar{z} .
$$

Using these, another calculation shows that

$$
\bar{\partial} \bar{\partial}^{*} \beta-\bar{\nabla}^{*} \bar{\nabla} \beta=e^{\psi} \Delta(\xi+\psi) \beta,
$$


and thus one has the identity

$$
\left\|\bar{\partial}^{*} \beta\right\|^{2}=\|\bar{\nabla} \beta\|^{2}+\left(e^{\psi} \Delta(\xi+\psi) \beta, \beta\right), \quad \beta \in \mathscr{A}_{0}^{0,1}(X) .
$$

Now,

$$
\Delta \xi=\Delta \varphi-\frac{1}{\tau} \Delta \tau+\sqrt{-1} \frac{\partial \tau \wedge \bar{\partial} \tau}{\tau^{2}}
$$

and formula (2.6) implies that

$$
\bar{\partial}_{\xi}^{*}(h d \bar{z})=\bar{\partial}_{\varphi}^{*}(h d \bar{z})-\frac{1}{\tau} \frac{\partial \tau}{\partial z} h .
$$

Substituting (2.8) and (2.9) into the identity (2.7), we obtain

$$
\begin{aligned}
\left\|\bar{\partial}_{\varphi}^{*} \beta\right\|_{\xi}^{2}=\|\bar{\nabla} \beta\|_{\xi}^{2}+ & \left(e^{\psi}\left\{\Delta(\varphi+\psi)-\frac{\Delta \tau}{\tau}+\frac{|\partial \tau|^{2}}{\tau^{2}}\right\} \beta, \beta\right)_{\xi} \\
& -\left\|\frac{\langle\partial \tau, \beta\rangle}{\tau}\right\|_{\xi}^{2}+2 \operatorname{Re}\left(\bar{\partial}_{\varphi}^{*} \beta, \frac{\langle\partial \tau, \beta\rangle}{\tau}\right)_{\xi} \\
= & \|\bar{\nabla} \beta\|_{\xi}^{2}+\left(e^{\psi}\left\{\Delta(\varphi+\psi)-\frac{\Delta \tau}{\tau}\right\} \beta, \beta\right)_{\xi}+2 \operatorname{Re}\left(\bar{\partial}_{\varphi}^{*} \beta, \frac{\langle\partial \tau, \beta\rangle}{\tau}\right)
\end{aligned}
$$

Substituting $e^{-\xi}=\tau e^{-\eta}$, we have the so-called twisted Bochner-Kodaira Identity:

$$
\begin{gathered}
\left\|\sqrt{\tau} \bar{\partial}_{\varphi}^{*} \beta\right\|_{\varphi}^{2}=\|\sqrt{\tau} \bar{\nabla} \beta\|_{\varphi}^{2}+\left(e^{\psi}\{\tau \Delta(\varphi+\psi)-\Delta \tau\} \beta, \beta\right)_{\varphi} \\
+2 \operatorname{Re}\left(\bar{\partial}_{\varphi}^{*} \beta,\langle\partial \tau, \beta\rangle\right)_{\varphi}
\end{gathered}
$$

The Cauchy-Schwarz inequality applied to the last term of (2.10) then shows that for any $\varepsilon>0$ we have

$$
\left(1+\varepsilon^{-1}\right)\left\|\sqrt{\tau} \bar{\partial}_{\varphi}^{*} \beta\right\|_{\varphi}^{2} \geq\left(e^{\psi}\left\{\tau \Delta(\varphi+\psi)-\Delta \tau-\varepsilon \frac{|\partial \tau|^{2}}{\tau}\right\} \beta, \beta\right)_{\varphi} .
$$

Letting $T f:=\bar{\partial}(\sqrt{\tau} f)$, we can rewrite $(2.11)$ as

$$
\left\|T^{*} \beta\right\|_{\varphi}^{2} \geq C_{\varepsilon}\left(e^{\psi}\left\{\tau \Delta(\varphi+\psi)-\Delta \tau-\varepsilon \frac{|\partial \tau|^{2}}{\tau}\right\} \beta, \beta\right)_{\varphi} .
$$

Suppose now that for some $\varepsilon>0$ there is a $\delta>0$ such that one has

$$
e^{\psi}\left(\tau \Delta(\varphi+\psi)-\Delta \tau-\varepsilon \frac{|\partial \tau|^{2}}{\tau}\right) \geq \delta
$$

The from (2.12) and the fact that smooth compactly supported (0,1)-forms are dense in the domain of $T^{*}$, we obtain

$$
\left\|T^{*} \beta\right\|_{\varphi}^{2} \geq C\|\beta\|_{\varphi}^{2}
$$


A standard Hilbert space argument yields a function $f$ such that

$$
T f=\alpha
$$

with the estimate

$$
\int_{X}|f|^{2} e^{-\varphi} d A_{g} \leq C\|\alpha\|_{\varphi}^{2}
$$

Letting $U=\sqrt{\tau} f$ completes the proof.

\section{Examples}

\section{The Euclidean plane}

Consider the Euclidean complex plane $(X, g)=\left(\mathbb{C},|d z|^{2}\right)$. The generalized Bergman space in this situation is

$$
\mathscr{B} \mathscr{F}^{2}=\left\{h \in \mathcal{O}(\mathbb{C}) ;\|h\|_{\varphi}^{2}:=\int_{\mathbb{C}}|h|^{2} e^{-\varphi} d m<+\infty\right\},
$$

where $d m$ is Lebesgue measure in the plane, and

$$
\mathfrak{B f}^{2}=\left\{\left(s_{\gamma}\right) \subset \mathbb{C} ;\left\|\left(s_{\gamma}\right)\right\|_{\varphi}^{2}:=\sum_{\gamma \in \Gamma}\left|s_{\gamma}\right|^{2} e^{-\varphi(\gamma)}<+\infty\right\} .
$$

The space $\mathscr{B} \mathscr{F}^{2}$ is sometimes called generalized Bargmann-Fock space. When $\varphi(z)=|z|^{2}$ we obtain the classical Bargmann-Fock space.

The plane is a parabolic Riemann surface. The Evans kernel in $\mathbb{C}$ is unique, up to an additive constant, and is given by $E(z, \zeta)=\log |z-\zeta|$ if we require $E(0,1)=0$. Thus $\rho_{z}(\zeta)=|z-\zeta|$ and the disks $D_{\sigma}(z)$ are simply the Euclidean disks $|z-\zeta|<\sigma$. A simple calculation shows that

$$
\sqrt{-1} \partial \rho_{z}(\zeta) \wedge \bar{\partial} \rho_{z}(\zeta)=\frac{1}{2} d \rho_{z}(\zeta) \wedge * d \rho_{z}(\zeta)=\frac{\sqrt{-1}}{4} d \zeta \wedge d \bar{\zeta}
$$

and thus the fundamental metric is just a multiple of the Euclidean metric.

The upper and lower densities are given by

$$
D_{f}^{+}(\Gamma)=\limsup _{r \rightarrow \infty} \sup _{z \in \mathbb{C}} \sum_{\Gamma \cap D_{r}(z)} \frac{f(|z-\gamma|)}{2 \Delta \varphi \int_{0}^{r} t f(t) d t}
$$

and

$$
D_{f}^{-}(\Gamma)=\liminf _{r \rightarrow \infty} \inf _{z \in \mathbb{C}} \sum_{\Gamma \cap D_{r}(z)} \frac{f(|z-\gamma|)}{2 \Delta \varphi \int_{0}^{r} t f(t) d t}
$$


If we choose as our locally integrable function $f$ the constant function, we recover the results of [1]. However, by making other choices, we can get other sufficient conditions that, although not necessary, might be of use in some applications.

For the sake of simplicity, we will consider in the following examples only the classical Bargmann-Fock space.

Example 3.1. (i) Let $f(t)=e^{-t}$. Then $\Gamma$ is interpolating if for all $r \gg 0$,

$$
\sup _{z \in \mathbb{C}} \sum_{\Gamma \cap D_{r}(z)} e^{-|z-\gamma|}<2
$$

and sampling if for all $r \gg 0$,

$$
\inf _{z \in \mathbb{C}} \sum_{\Gamma \cap D_{r}(z)} e^{-|z-\gamma|}>2 .
$$

Integration by parts, together with a standard argument shows that $\Gamma$ is interpolating if

$$
\sup _{z \in \mathbb{C}} \int_{0}^{\infty} \#\left(\Gamma \cap D_{s}(z)\right) \frac{d s}{e^{s}}<2
$$

and sampling if

$$
\inf _{z \in \mathbb{C}} \int_{0}^{\infty} \#\left(\Gamma \cap D_{s}(z)\right) \frac{d s}{e^{s}}>2 .
$$

(ii) Let $f_{a}:=\mathbf{1}_{[0, a]}$. We then obtain:

If $a>1 / \sqrt{2}$ and every disk of radius $a$ contains at most one member of $\Gamma$, then $\Gamma$ is interpolating.

If $a<1 / \sqrt{2}$ and every disk of radius $a$ contains at least one member of $\Gamma$, then $\Gamma$ is sampling.

\section{The disk}

The unit disk $\mathbb{D}$ is a hyperbolic Riemann surface. Its Green's function is

$$
E(z, \zeta)=\log \left|\phi_{z}(\zeta)\right|, \quad \text { where } \quad \phi_{z}(\zeta)=\frac{z-\zeta}{1-\bar{z} \zeta}
$$

is the standard involution. Thus $\rho_{z}(\zeta)=\left|\phi_{z}(\zeta)\right|$ and the disks $D_{\sigma}(z)$ are the well-known pseudo-hyperbolic disks. Standard calculations show that

$$
\left|d \rho_{z}(\zeta)\right|^{2}=\left|\frac{1-|z|^{2}}{(1-\bar{z} \zeta)^{2}}\right|^{2}=\frac{\left(1-\rho_{z}(\zeta)^{2}\right)^{2}}{\left(1-|\zeta|^{2}\right)^{2}},
$$

so we have $\nu(\zeta)=-\log 4+\log \left(1-|\zeta|^{2}\right)^{2}$. 
We endow $\mathbb{D}$ with the metric $g=\left(1-|z|^{2}\right)^{-1}|d z|^{2}$, and thus obtain

$$
\tau_{\psi}=\frac{1}{4}\left(1-|z|^{2}\right), \quad \mathfrak{D}_{\psi}^{2}=\frac{1}{4}|z|^{2}
$$

and

$$
\begin{aligned}
e^{\nu} \Theta_{\psi} & =\frac{1}{4}\left(1-|z|^{2}\right)^{2}\left(\Delta \psi-\tau_{\psi}^{-1} \Delta \tau_{\psi}^{-1}\right) \\
& =\frac{\left(1-|z|^{2}\right)^{2}}{4}\left(-\left(1-|z|^{2}\right)^{-2}+\left(1+|z|^{2}\right)^{-1}\right)=\frac{-|z|^{2}}{4} .
\end{aligned}
$$

We also have

$$
A_{g}\left(D_{\sigma}(\gamma)\right)=C_{\sigma}\left(1-|\gamma|^{2}\right)
$$

Thus our Hilbert spaces are

$$
\mathscr{B}_{\varphi}^{2}:=\left\{h \in \mathcal{O}(\mathbb{D}) ; \int_{\mathbb{D}}|h|^{2} e^{-\varphi} \frac{d m}{\left(1-|z|^{2}\right)}<+\infty\right\}
$$

and

$$
\mathfrak{B}_{\varphi}^{2}:=\left\{\left(s_{\gamma}\right) ; \sum_{\gamma \in \Gamma}\left|s_{\gamma}\right|^{2} e^{-\varphi(\gamma)}\left(1-|\gamma|^{2}\right)<+\infty\right\} .
$$

Finally, we let

$$
\varphi:=\varphi_{o}-\log \left(1-|z|^{2}\right), \quad \Delta \varphi_{o}>0
$$

Observe that

$$
\Delta \varphi+\Theta_{\psi}=\Delta \varphi_{o}+\frac{1}{\left(1-|z|^{2}\right)^{2}}-\frac{|z|^{2}}{4} \geq \Delta \varphi_{o}>0 .
$$

Thus the densities are given by

$$
\begin{aligned}
D_{f}^{+}(\Gamma)= & \limsup _{r \rightarrow 1} \sup _{z \in \mathbb{D}} \sum_{\rho_{z}(\gamma)<r} \frac{f\left(\rho_{z}(\gamma)\right)\left(1-\rho_{z}(\gamma)^{2}\right)^{2}}{\left(\left(1-|z|^{2}\right)^{2} \Delta \varphi_{o}(z)+\left(1-|z|^{2}\right)\right) \int_{0}^{r} t f(t) d t} \\
& \text { and } \\
D_{f}^{-}(\Gamma)= & \liminf _{r \rightarrow 1} \inf _{z \in \mathbb{D}} \sum_{\rho_{z}(\gamma)<r} \frac{f\left(\rho_{z}(\gamma)\right)\left(1-\rho_{z}(\gamma)^{2}\right)^{2}}{\left(\left(1-|z|^{2}\right)^{2} \Delta \varphi_{o}(z)+\left(1-|z|^{2}\right)\right) \int_{0}^{r} t f(t) d t}
\end{aligned}
$$

If we take

$$
f(t)=\frac{-\log t}{\left(1-t^{2}\right)^{2}} \mathbf{1}_{\left[\frac{1}{2}, 1\right)},
$$

we find that Theorems 1 and 2 recover the results from [1].

Again for the sake of illustration we will consider below only the classical unweighted Bergman space, which is obtained by setting $\varphi=-\log \left(1-|z|^{2}\right)$. 
Example 3.2. (i) Letting $f=1$, we see that $\Gamma$ is interpolating if

$$
\sup _{z \in \mathbb{D}} \sum\left(1-\rho_{z}(\gamma)^{2}\right)^{2}<1
$$

and sampling if

$$
\inf _{z \in \mathbb{D}} \sum\left(1-\rho_{z}(\gamma)^{2}\right)^{2}>1
$$

(ii) Letting $f(t)=\left(1-t^{2}\right)^{-2}$, we see that $\Gamma$ is interpolating if

$$
\limsup _{r \rightarrow 1} \sup _{z \in \mathbb{D}} \frac{\#\left(\Gamma \cap D_{r}(z)\right)}{A_{\text {hyp }}\left(D_{r}(z)\right)}<1
$$

and sampling if

$$
\liminf _{r \rightarrow 1} \inf _{z \in \mathbb{D}} \frac{\#\left(\Gamma \cap D_{r}(z)\right)}{A_{\text {hyp }}\left(D_{r}(z)\right)}>1
$$

where

$$
A_{\text {hyp }}\left(D_{r}(z)\right)=\frac{1}{2 \pi} \int_{D_{r}(z)} \frac{d m(z)}{\left(1-|z|^{2}\right)^{2}}
$$

denotes hyperbolic area of $D_{r}(z)$.

(iii) Let $f_{a}:=\mathbf{1}_{[0, a]}$. We then obtain:

If $\delta>\frac{1}{\sqrt{2}}$ and $\Gamma$ has at most one point in every disk of radius $\delta$, then $\Gamma$ is interpolating.

If $\delta<\frac{1}{\sqrt{2}}$ and every disk of radius $\delta$ contains at least one member of $\Gamma$, then $\Gamma$ is sampling.

\section{Finite Riemann surfaces}

\section{Definition and construction of finite Riemann surfaces}

Recall that a finite Riemann surface is a two dimensional compact manifold with boundary, possibly with a finite number of points removed.

There are two types of finite Riemann surfaces. One type has boundary having only punctures and no one dimensional components, while the other type also has at least one smooth codimension-1 boundary component. The first type of is always parabolic (unless it has no punctures, in which case it is compact) while the second type is always hyperbolic.

An alternate description of a finite Riemann surface $X$ can be given as follows: $X$ is a (not necessarily compact) manifold with compact boundary, and in addition $X$ can be decomposed as

$$
X=X_{\text {core }} \cup \bigcup_{j=1}^{N} U_{j},
$$


where $X_{\text {core }}$ is a compact manifold with smooth boundary, and each $U_{j}$ is biholomorphic to a punctured disk whose outer boundary is one of the smooth boundary curves of $X_{\text {core }}$. (Of course, $X_{\text {core }}$ may have some other boundary components that do not meet one of the $U_{j}$.) The $U_{j}$ correspond to the punctures.

While every finite Riemann surface with no one dimensional boundary is obtained from a compact Riemann surface by removal of a finite number of points, there is an almost equally simple way to construct hyperbolic finite Riemann surfaces; simply take a compact Riemann surface and remove a finite number of smooth Jordan curves so that the resulting surface as two components. Then either component is a finite Riemann surface, and one can further remove any finite number of points.

In fact, all finite Riemann surfaces are of this type. Indeed, we can fill in the punctures complex analytically (since they are just punctured disks) to obtain a compact Riemann surface with boundary

$$
\tilde{X}=X_{\text {core }} \cup \bigcup_{j=1}^{N} \overline{U_{j}},
$$

and then form the so-called double of $\tilde{X}$. For more on this well-known construction see, for example, [7].

\section{Analytic-geometric properties of finite Riemann surfaces}

Theorem 4.1. Let $X$ be a finite Riemann surface with extremal fundamental solution $E$. Then for each sufficiently small $\sigma \in\left(0, R_{X}\right)$ there is a constant $C=C_{\sigma}$ such that for all $z \in X$ and all $\zeta \in D_{\sigma}(z)$ the following estimate holds.

$$
\frac{1}{C} \leq e^{\nu(\zeta)}\left|\partial \rho_{z}(\zeta)\right|^{2} \leq C
$$

Remark. As we have already pointed out, locally one has

$$
E(z, \zeta)=\log |\zeta-z|+h(z, \zeta)
$$

and thus $\rho_{z}(\zeta)=|z-\zeta| e^{h(z, \zeta)}$. Differentiation then gives

$$
\partial \rho_{z}(\zeta)=\frac{\overline{\zeta-z}}{|\zeta-z|} e^{h(z, \zeta)}\left(\frac{1}{2}+(z-\zeta) \partial_{\zeta} h(z, \zeta)\right),
$$

so that $4 e^{-\nu(\zeta)}=e^{2 h(\zeta, \zeta)}$ and

$$
e^{\nu(\zeta)}\left|\partial \rho_{z}(\zeta)\right|^{2}=e^{2(h(z, \zeta)-h(\zeta, \zeta))}\left|1+2(\zeta-z) \frac{\partial h(z, \zeta)}{\partial \zeta}\right|^{2} .
$$

In particular, the right hand side of (4.2) is well defined, since this is the case for the left hand side. 
Proof of theorem 4.1. We shall break up the proof into the hyperbolic and parabolic case.

(The case of bordered Riemann surfaces.) We realize $X$ as an open subset of its double $Y$. Since $\bar{X}=X \cup \partial X$ is compact, it suffices to bound the right hand side of (4.2) in a set $U \cap X$, where $U$ is a coordinate chart in $Y$. For coordinate charts whose closure lies in the interior $X$, it is clear that this can be done. Indeed, if $U \subset \subset X$ and $z, \zeta \in U$, then $h$ is a smooth function that is harmonic in each variable separately, and $\rho_{z}(\zeta) \asymp|\zeta-z|$ uniformly on $U$. Thus by taking $\sigma$ sufficiently small, we obtain the estimate (4.1) for all $z \in U$ and $\zeta \in D_{\sigma}(z)$. We thus restrict our attention to the boundary.

There are two types of boundary points; zero dimensional and one dimensional. However, the Green's function ignores isolated zero dimensional boundary components, since they have capacity zero. (In particular, the distance $\rho_{z}$ fails to be proper when there are punctures.) Thus we may assume that there are no punctures.

Let $U \subset Y$ be a coordinate neighborhood of a boundary point $x \in \partial X$. By taking $U$ sufficiently small, we may assume that $U$ is the unit disk in the plane, that $U \cap X$ lies in the upper half plane and that $\partial X$ lies on the real line. It follows that the Green's function is given by

$$
E(z, \zeta)=\log |z-\zeta|-\log |\bar{z}-\zeta|+F(z, \zeta),
$$

where $F(z, \zeta)$ is smooth and harmonic in each variable on a large open set containing the closure of $U$. Indeed, the Green's function for the upper half plane is $\log |z-\zeta|-\log |\bar{z}-\zeta|$. The regularity of $F$ then follows from the construction of Green's functions on finite Riemann surfaces using harmonic differentials on the double. (See $[7, \S 4.2]$.) It follows that in $U$,

$$
2 \frac{\partial h(z, \zeta)}{\partial \zeta}=-\frac{1}{\bar{z}-\zeta}+2 \frac{\partial F(z, \zeta)}{\partial \zeta} \quad \text { and } \quad \rho_{z}(\zeta) \geq C \frac{|z-\zeta|}{|\bar{z}-\zeta|}
$$

Thus

$$
\begin{aligned}
\left|2(\zeta-z) \frac{\partial h(z, \zeta)}{\partial \zeta}\right| & \leq \frac{|z-\zeta|}{|\bar{z}-\zeta|}+2|z-\zeta|\left|\frac{\partial F(z, \zeta)}{\partial \zeta}\right| \\
& \leq C \frac{|z-\zeta|}{|\bar{z}-\zeta|} \leq C^{\prime} \rho_{z}(\zeta)
\end{aligned}
$$

where the constant $C^{\prime}$ depends only on the neighborhood $U$. The proof in the hyperbolic case is thus complete.

(The case of compact Riemann surfaces with punctures.) Let $E$ be the chosen extremal fundamental solution of $X$. Fix $p \in X$ and choose $r$ so large that the set $X-D_{r}(z)$ is a union of punctured disks $U_{1}, \ldots, U_{N}$. We may think of each $U_{j}$ as sitting in $\mathbb{C}$, with the puncture at the origin. 
Since $D_{r}(z) \subset \subset X$, each $x \in \overline{D_{r}(z)}$ has a neighborhood $U$ for which the expression (4.2) is bounded above and below by positive constants, depending only on $U$, whenever $\rho_{z}(\zeta)<\sigma$ for some sufficiently small $\sigma$ again depending only on $U$. Indeed, in any such neighborhood the function $h$ is very regular, and $\rho_{z}(\zeta)$ is uniformly comparable to $|z-\zeta|$.

Next, for all but one of the punctures, our kernel is again regular by our choices. (See the remark in the first paragraph of Section 2.) Thus we may focus on the one puncture where we have a singularity, which we call $U_{j}$.

For $z, \zeta \in U_{j}$, the Evans kernel has the form

$$
E(z, \zeta)=\log |z-\zeta|-\log |\zeta|+F(z, \zeta)
$$

where $F(z, \zeta)$ is smooth across the origin (see [3]). Indeed, using the method of constructing harmonic differentials with prescribed singularities (see $[7$, $\S 2.7])$ we can construct a function with the right singularities, defined everywhere on $\bar{X}$. Such a function clearly can be written in the form (4.3) near the puncture. Thus by the uniqueness of the Evans kernel for a surface with a single puncture, this function must differ from $E$ by a constant.

It follows that in $U$,

$$
h(z, \zeta)-h(\zeta, \zeta) \sim 1, \quad 2 \frac{\partial h(z, \zeta)}{\partial \zeta}=-\frac{1}{\zeta}+\frac{\partial F(z, \zeta)}{\partial \zeta}
$$

and

$$
\rho_{z}(\zeta) \geq C \frac{|z-\zeta|}{|\zeta|}
$$

Thus

$$
\begin{aligned}
\left|2(\zeta-z) \frac{\partial h(z, \zeta)}{\partial \zeta}\right| & \leq \frac{|z-\zeta|}{|\zeta|}+2|z-\zeta|\left|\frac{\partial F(z, \zeta)}{\partial \zeta}\right| \\
& \leq C \frac{|z-\zeta|}{|\zeta|} \\
& \leq C^{\prime} \rho_{z}(\zeta),
\end{aligned}
$$

where again the constant $C^{\prime}$ depends only on the neighborhood $U$. The proof of Theorem 4.1 is thus complete.

Proposition 4.2. Let $X$ be a finite Riemann surface. Then there exists a constant $C$ such that, for sufficiently small $\sigma>0$ and all $z \in X$,

$$
\begin{aligned}
& \sup _{w \in D_{\sigma}(z)} \exp \left(\frac{4}{\pi} \int_{D_{2 \sigma}(z)}-G(w, \zeta) e^{-\nu(\zeta)}\right) \\
& \leq C \inf _{w \in D_{\sigma}(z)} \exp \left(\frac{4}{\pi} \int_{D_{2 \sigma}(z)}-G(w, \zeta) e^{-\nu(\zeta)}\right)<+\infty
\end{aligned}
$$

where $G$ is the Green's function for the domain $D_{2 \sigma}(z)$. 
Sketch of proof. Once again we can use compactness properties of finite surfaces. The finiteness of the integrals in question is easy, since extremal fundamental solutions have only a logarithmic singularity, and are thus locally integrable. Thus we restrict ourselves to estimating near the boundary.

The local analysis used in the proof of Theorem 4.1 shows that, near the boundary, the disks $D_{\sigma}(z)$ are simply connected and that the metric $e^{-\nu}$ is equivalent to the Poincaré metric of the disk in the hyperbolic case, and the metric $|z|^{-2}|d z|^{2}$ in the parabolic case.

The hyperbolic case follows from the fact that the Green's function $G(w, \zeta)$ is comparable to the Green's function of the disk. In the parabolic case it is easier to work with the complement of the unit disk rather than the punctured disk. Then the metric $e^{-\nu}$ is comparable to the Euclidean metric, the Green's function $G(w, \zeta)$ is comparable to the Green's function of the plane, and the necessary estimate follows as in the Euclidean case. This completes the sketch of proof.

Lemma 4.3. Let $X$ be a finite Riemann surface. Let $\sigma>0$ be a fixed, sufficiently small constant. If $\varphi$ is a function for which $e^{\nu} \Delta \varphi$ is bounded above and below by positive constants, then there is a constant $C=C_{\sigma}$ such that, for all $z \in X$ and all $w \in D_{\sigma}(z)$,

$$
\exp \left(\frac{4}{\pi} \int_{D_{2 \sigma}(z)}-G(w, \zeta) \Delta \varphi(\zeta)\right) \leq C
$$

Proof. By Theorem 4.1, Proposition 4.2 and the boundedness of $\Delta \varphi$, it suffices to prove the result when $\Delta \varphi(\zeta)=d \rho_{z}(\zeta) \wedge * d \rho_{z}(\zeta)$ and $w=z$. In this case, it is easy to show that the integral is equal to $8 \sigma^{2}$.

The next result we will need is a global version of the Cauchy estimates on a Riemann surface with Riemannian metric.

Proposition 4.4. Let $X$ be a finite Riemann surface and let $g$ be a conformal metric for $X$. Then for every $\sigma \in\left[0, R_{X}\right)$ and $\varepsilon>0$ there exists a constant $C_{\varepsilon, \sigma}$ such that for any $x \in X$ the following Cauchy estimates hold.

$$
\sup _{D_{\varepsilon}(x)}|h|^{2} \leq C_{\varepsilon, \sigma} \int_{D_{\sigma}(x)}|h|^{2} d A_{g}
$$

and

$$
\sup _{D_{\varepsilon}(x)}\left|\partial \rho_{x}\right|^{-2}\left|h^{\prime}\right|^{2} \leq C_{\varepsilon, \sigma} \int_{D_{\sigma}(x)}|h|^{2} d A_{g}
$$

To establish Proposition 4.4, we need the following lemma. 
Lemma 4.5. Let $X$ be a finite Riemann surface. Then for every $x \in X$ there exists a function $K^{x}: X \times X \rightarrow \mathbb{R}$ such that the following hold for any $\sigma \in\left[0, R_{X}\right)$ :

1. In the sense of distributions, $\Delta_{z} K^{x}(z, \zeta)=\frac{\pi}{2} \delta_{z}(\zeta)$ for all $z, \zeta \in D_{\sigma}(x)$.

2. For every $\varepsilon<\sigma / 4$ there exists a constant $C_{\varepsilon, \sigma}$ such that for any $x \in X$ the following estimates hold:

$$
\begin{aligned}
& \sup _{z \in D_{\varepsilon}(x)} \int_{V_{\sigma}(x)} e^{\psi}\left|\frac{\partial \rho_{x}}{\partial \zeta} \frac{\partial K^{x}(z, \zeta)}{\partial \zeta}\right|^{2} \leq C_{\varepsilon, \sigma} \\
& \sup _{z \in D_{\varepsilon}(x)}\left|\partial \rho_{x}(z)\right|^{-2} \int_{V_{\sigma}(x)} e^{\psi}\left|\partial \rho_{x}\right|^{2}\left|\frac{\partial^{2} K^{x}(z, \zeta)}{\partial z \partial \zeta}\right|^{2} \leq C_{\varepsilon, \sigma}
\end{aligned}
$$

Here $V_{\sigma}(x):=D_{\sigma}(x)-D_{\sigma / 2}(x)$.

Sketch of proof. In the case of a bordered Riemann surface with a finite number of punctures, one can find a function $K^{x}$ that does not depend on the point $x$. This is done as follows. Let $Y$ be the double of $X$, and fix any smooth distance function on $Y$. We let $X_{\varepsilon}$ be the set of all $x \in Y$ that are a distance less than $\varepsilon$ from $X$. For $\varepsilon$ sufficiently small, $X_{\varepsilon}-X$ is a finite collection of annuli whose inner boundaries form the boundary of $X$. We may take for our Cauchy-Green kernel the Green's function of $X_{\varepsilon}$. We leave it to the reader to check that the relevant estimates hold.

In the case of an $N$-punctured compact Riemann surface, one decomposes $X$ as

$$
X=X_{\text {core }} \cup \bigcup_{j=1}^{N} U_{j},
$$

where $X_{\text {core }}$ is a bordered Riemann surface, and each $U_{j}$ is a neighborhood of a puncture biholomorphic to the punctured disk. Each surface in the union has a Cauchy-Green kernel by the construction in the bordered Riemann surface case, and thus we are done.

Proof of Proposition 4.4. Let $f \in \mathcal{C}_{0}^{\infty}\left(D_{\sigma}(x)\right)$ and write $K_{z}^{x}(\zeta)=K^{x}(z, \zeta)$. Applying formula $(2.1)$ with $h(\zeta)=K_{z}^{x}(\zeta)$, we obtain

$$
\frac{\pi}{2} f(z)=\int_{D_{\sigma}(x)} K_{z}^{x} d \bar{\partial} f=\int_{D_{\sigma}(x)} \bar{\partial} f \wedge \partial K_{z}^{x} .
$$

Now let $\varepsilon<\sigma / 4$ and let $\chi \in \mathcal{C}_{0}^{\infty}([0,3 \sigma / 4))$ be such that

$$
\chi \mid[0, \sigma / 2] \equiv 1 \quad \text { and } \quad \sup \left|\chi^{\prime}\right| \leq \frac{5}{\sigma}
$$


If $h \in \mathcal{O}\left(D_{\sigma}(x)\right)$, then with $z \in D_{\varepsilon}(x)$ we have

$$
h(z)=\int_{D_{\sigma}(x)} h \chi^{\prime}\left(\rho_{x}\right) \bar{\partial} \rho_{x} \wedge \partial K_{z}^{x} .
$$

An application of the Cauchy-Schwarz inequality and the estimate (4.8) gives the inequality (4.6), while differentiation of (4.10) followed by an application of the Cauchy-Schwarz inequality and the estimate (4.9) gives inequality (4.7).

Remark. Note that were it not for the requirement that $C_{\varepsilon, \sigma}$ be independent of $x$, Proposition 4.4 would follow without (4.8) and (4.9).

\section{Discrete subsets in finite Riemann surfaces}

Let $X$ be an open Riemann surface. Our work on sampling and interpolation sequences requires the notion of the separation of a sequence. For a measurable subset $A \subset X$, let

$$
D_{r}(A)=\left\{w \in X ; w \in D_{r}(a) \text { for some } a \in A\right\} .
$$

We define two separation conditions on a sequence $\Gamma$, both of which are given in terms of the distance induced by the extremal fundamental solution.

Definition 4.6. Let $\Gamma \subset X$ be a discrete set.

1. The separation constant of $\Gamma$ is the number

$$
\sigma(\Gamma):=\sup \left\{r ; D_{r}(\gamma) \cap D_{r}\left(\gamma^{\prime}\right)=\emptyset\right\}
$$

and say that $\Gamma$ is uniformly separated if $\sigma(\Gamma)>0$.

2. We say $\Gamma$ is sparse if there is a positive constant $N_{r, \varepsilon}$, depending only on $0<r, \varepsilon<R_{X}$, such that the number of points of $\Gamma$ lying in the set $D_{r}\left(D_{\varepsilon}(z)\right)$ is at most $N_{r, \varepsilon}$ for all $z \in X$.

In both the complex plane and the unit disk, the triangle inequality allows one to estimate the diameter of a set $D_{\varepsilon}\left(D_{r}(a)\right)$ in terms of $\varepsilon$ and $r$, and thus show that a uniformly separated sequence is sparse.

Such an diameter estimate can always be found if it is allowed to depend on the base point $a$. This situation can be made uniform when $X$ is a finite Riemann surface. As in the proofs of Theorem 4.1 and Propositions 4.2 and 4.4 , we can take advantage of the compactness in the picture. In particular, we have uniform estimates if we have them in neighborhoods of the boundary. But on the boundary, the potential theory of $X$ is either like that (near the boundary) of the upper half plane or (near infinity) of the plane, where we know, from triangle inequalities in those cases, that the needed estimates hold. We thus have the following proposition. 
Proposition 4.7. In a finite Riemann surface $X$ every uniformly separated sequence is sparse.

Remark. We do not know whether Proposition 4.7 holds if one removes the finiteness condition.

\section{Compact Riemann surfaces}

\section{Cohomological criterion for interpolation and sampling}

Let $X$ then be a compact Riemann surface and let $V \rightarrow X$ be a holomorphic line bundle. We denote by $V_{x}$ the fiber of $V$ over $x \in X$. Then $\Gamma$ is interpolating if and only if the evaluation map

$$
H^{0}(X, L) \ni s \mapsto \sum_{\gamma \in \Gamma} s(\gamma) \in \bigoplus_{\gamma \in \Gamma} V_{\gamma}
$$

is surjective, and sampling if and only if (5.1) is injective.

Let $\Lambda$ be the line bundle corresponding to the effective divisor $\Gamma$. One can understand the situation completely using the short exact sequence of sheaves

$$
0 \rightarrow \mathcal{O}_{X}\left(L \otimes \Lambda^{*}\right) \rightarrow \mathcal{O}_{X}(L) \rightarrow \bigoplus_{\gamma \in \Gamma} \mathcal{V}_{\gamma} \rightarrow 0
$$

where $\mathcal{V}_{\gamma}(U)=V_{\gamma}$ if $\gamma \in U$ and $\mathcal{V}_{\gamma}(U)=0$ if $\gamma \notin U$. Passing to the long exact sequence, we have that

$$
\begin{aligned}
0 \rightarrow H^{0}\left(X, L \otimes \Lambda^{*}\right) \stackrel{i_{0}}{\longrightarrow} H^{0}(X, L) \stackrel{e_{\Gamma}}{\longrightarrow} \bigoplus_{\gamma \in \Gamma} V_{\gamma} \stackrel{\delta_{0}}{\longrightarrow} H^{1}\left(X, L \otimes \Lambda^{*}\right) \\
\stackrel{i_{1}}{\longrightarrow} H^{1}(X, L) \rightarrow \ldots
\end{aligned}
$$

We see that $e$ is injective if and only if Image $\left(i_{0}\right)=\{0\}$ and surjective if and only if $i_{1}$ is injective, i.e., Image $\left(\delta_{0}\right)=\{0\}$. We then have the following proposition.

Proposition 5.1. Let $X$ be a compact Riemann surface of genus $g, \Gamma \subset X$ a finite subset and $L \rightarrow X$ a holomorphic line bundle.

1. If $\# \Gamma<\operatorname{deg}(L)+2-2 g$, then $\Gamma$ is interpolating.

2. If $\# \Gamma>\operatorname{deg}(L)$, then $\Gamma$ is sampling.

Proof. To establish 1 , note that by Serre duality, $h^{1}\left(X, L \otimes \Lambda^{*}\right)=h^{0}\left(X, K_{X} \otimes\right.$ $\left.\Lambda \otimes L^{*}\right)$, and the latter vanishes if

$$
\# \Gamma+2 g-2-\operatorname{deg}(L)=\operatorname{deg}\left(K_{X} \otimes \Lambda \otimes L^{*}\right)<0 .
$$

Similarly, if $\operatorname{deg}(L)-\# \Gamma=\operatorname{deg}\left(L \otimes \Lambda^{*}\right)<0$, then $h^{0}\left(X, L \otimes \Lambda^{*}\right)=0$. 


\section{Analytic proof of Proposition 5.1(1)}

Part (1) of Proposition 5.1 can also be proved using Theorem 2.6. Because it is similar to the proof of our main interpolation theorem, we sketch this method here.

Let $\sum v_{\gamma} \in \bigoplus V_{\gamma}$. First, observe that there is a smooth section $\eta$ of $L$ such that $\eta(\gamma)=v_{\gamma}$ for all $\gamma \in \Gamma$. In fact, by the usual cutoff method, we can take $\eta$ supported near $\Gamma$ and holomorphic in a neighborhood of $\Gamma$.

Fix a conformal metric $e^{-\psi}|d z|^{2}$ on $X$. Let $\tau$ be the canonical section of $\Lambda$ corresponding to the divisor $\Gamma$. By the degree hypothesis, there is a metric $e^{-\varphi}$ for the line bundle $L \otimes \Lambda^{*}$ such that the curvature $\sqrt{-1} \partial \bar{\partial}(\varphi+\psi)$ of $L \otimes \Lambda^{*} \otimes K_{X}^{*}$ is strictly positive on $X$. Then $e^{-\varphi} /|\tau|^{2}$ is a singular metric for $L$ such that the curvature current of $e^{-(\varphi+\psi)} /|\tau|^{2}$ is still strictly positive on $X$. Moreover, since $\eta$ is holomorphic in a neighborhood of $\Gamma$, we have $\int_{X}|\bar{\partial} \eta|^{2}|\tau|^{-2} e^{-\varphi}<+\infty$. By Hörmander's Theorem there is a section $u$ of $L$ such that $\bar{\partial} u=\bar{\partial} \eta$ and $\int_{X}|u|^{2}|\tau|^{-2} e^{-(\varphi+\psi)}<+\infty$. But since $\tau$ vanishes on $\Gamma$, so does $u$. Thus $\sigma=\eta-u$ is holomorphic and solves the interpolation problem.

Remark. We note that if $e^{-\varphi}$ is a metric for a holomorphic line bundle $L$, then

$$
\operatorname{deg}(L)=\frac{1}{4 \pi} \int_{X} \Delta \varphi
$$

This fact shows the resemblance between Proposition 5.1 and our main theorems.

\section{Functions and singular weights}

\section{A local construction of a holomorphic function}

In the proofs of Theorems 1 and 2 we will need, for each $\gamma \in \Gamma$, a holomorphic function defined in a neighborhood of $\gamma$ and satisfying certain global estimates. For reasons that will become clear later, the size of this neighborhood cannot be taken too small. As a consequence, we must overcome certain difficulties presented by the topology of the neighborhood.

Lemma 6.1. Let $X$ be a finite open Riemann surface. Assume $e^{\nu} \Delta \varphi$ is bounded above and below by positive constants. Let $\Gamma$ be a uniformly separated sequence. Then there exists a constant $C=C_{\Gamma}>0$ and, for each $\gamma \in \Gamma$, a holomorphic function $F_{\gamma} \in \mathcal{O}\left(D_{\sigma}(\gamma)\right)$ such that $F_{\gamma}(\gamma)=0$ and for all $z \in D_{\sigma}(\gamma)$,

$$
\frac{1}{C} e^{-\varphi(\gamma)} \leq\left|e^{-\varphi+2 F_{\gamma}}\right| \leq C e^{-\varphi(\gamma)}
$$


Proof. Let $G$ be the Green's function for the domain $D_{2 \sigma}(\gamma)$. Consider the function

$$
T_{\gamma}(z):=\frac{1}{\pi} \int_{D_{2 \sigma}(\gamma)}-G(z, \zeta) \Delta \varphi(\zeta) .
$$

By Green's formula, we have that

$$
2 T_{\gamma}(z)=-\varphi(z)+\frac{1}{2 \pi} \int_{S_{2 \sigma}(\gamma)} \varphi(\zeta) * d_{\zeta} G(z, \zeta) .
$$

We claim that the harmonic function

$$
h_{\gamma}:=\frac{1}{2 \pi} \int_{S_{2 \sigma}(\gamma)} \varphi(\zeta) * d_{\zeta} G(z, \zeta)
$$

has a harmonic conjugate, i.e., it is the real part of a holomorphic function. Indeed, if $\mathcal{C}$ is a Jordan curve in $D_{r}(\gamma)$, then

$$
\int_{\mathcal{C}} * d h_{\gamma}(z)=\frac{1}{2 \pi} \int_{S_{2 \sigma}(\gamma)} \varphi(\zeta) * d_{\zeta}\left(\int_{\mathcal{C}} * d_{z} G(z, \zeta)\right) .
$$

Since $S_{2 \sigma}(\gamma) \cap \mathcal{C}=\emptyset$, the function $z \mapsto G(z, \zeta)$ is harmonic and thus $* d_{z} G(z, \zeta)$ is a closed form. It follows that the term in the parentheses on the right hand side of (6.2) depends only on the homology class $[\mathcal{C}] \in H_{1}(X, \mathbb{Z})$. Since $H_{1}(X, \mathbb{Z})$ is discrete and $* d_{z} G(z, \zeta)$ is continuous in $\zeta$, we see that the right hand side of (6.2) vanishes, as claimed.

Let

$$
H_{\gamma}:=h_{\gamma}+\sqrt{-1} \int_{\gamma}^{z} * d h_{\gamma}
$$

be the holomorphic function whose real part is $h_{\gamma}$, and let $F_{\gamma}:=H_{\gamma}-H_{\gamma}(\gamma)$. We have

$$
\left|\varphi(\gamma)-\varphi(z)+2 \operatorname{Re} F_{\gamma}(z)\right|=2\left|T_{\gamma}(\gamma)-T_{\gamma}(z)\right| \leq 2\left|T_{\gamma}(\gamma)\right|+\left|T_{\gamma}(z)\right| .
$$

Taking exponentials and applying Lemma 4.3 completes the proof.

\section{A function with poles along $\Gamma$}

For $z, \zeta \in X$ and $r<R_{X}$, let

$$
\begin{aligned}
I(\zeta, z) & =\int_{X} \xi_{r}(\zeta, w) E(w, z) e^{-\nu(w)} \sqrt{-1} d w \wedge d \bar{w} \\
& =\frac{1}{c_{r}} \int_{a}^{r} t f(t)\left(\int_{S_{t}(\zeta)} E(w, z) * d E_{\zeta}(w)\right) d t
\end{aligned}
$$


Since $E$ is a fundamental solution to the Laplacian,

$$
e^{\nu(z)} \Delta_{z} I(\zeta, z)=\frac{\pi}{2} \int_{X} \xi_{r}(\zeta, w) \delta_{z}(w)=\frac{\pi}{2} \xi_{r}(\zeta, z)
$$

Next it follows from $(2.5)$ that, since $E(\cdot, z)$ is subharmonic, $E(\zeta, z) \leq I(\zeta, z)$ and, since $E(\cdot, z)$ is harmonic in the region $\left\{w \in X: \rho_{\zeta}(w)>r\right\}, E(\zeta, z)=$ $I(\zeta, z)$ if $\rho_{z}(\zeta)>r$. Moreover, in view of (2.2), an application of (2.3) shows that

$$
\frac{1}{2 \pi} \int_{S_{t}(\zeta)} E(w, z) * d E_{\zeta}(w)=E(z, \zeta)-\mathbf{1}_{D_{t}(z)}(\zeta)(E(z, \zeta)-\log t)
$$

We see that

$$
I(\zeta, z)=\frac{2 \pi}{c_{r}}\left(\log \left(\rho_{z}(\zeta)\right) \int_{0}^{\rho_{z}(\zeta)} t f(t) d t+\int_{\rho_{z}(\zeta)}^{r} t f(t) \log t d t\right)
$$

if $\rho_{z}(\zeta)<r$. Note that

$$
\left|\frac{1}{c_{r}} \int_{\rho_{z}(\zeta)}^{r} t f(t) \log (t) d t\right| \leq D_{r}
$$

where $D_{r}$ depends only on $r$. We then have

$$
|I(\zeta, z)| \leq K_{r} \rho_{z}(\zeta)\left|\log \left(\rho_{z}(\zeta)\right)\right|+D_{r}
$$

for all $z, \zeta \in X$ satisfying $\rho_{z}(\zeta)<r$. Since the expression on the right hand side is bounded by a constant that depends only on $r$, we have

$$
|I(\zeta, z)| \leq C_{r}
$$

whenever $\rho_{z}(\zeta)<r$.

Let $\Gamma$ be a discrete sequence. We define the function

$$
v_{r}(z)=\sum_{\gamma \in \Gamma}(E(\gamma, z)-I(\gamma, z)) .
$$

By the preceding remarks, $v_{r}(z) \leq 0$ and

$$
v_{r}(z)=\sum_{\gamma \in \Gamma \cap D_{r}(z)}(E(\gamma, z)-I(\gamma, z)) .
$$

Moreover,

$$
e^{\nu} \Delta v_{r}=\frac{\pi}{2} \sum_{\gamma \in \Gamma}\left(e^{\nu} \delta_{\gamma}-\xi_{r}(\gamma, \cdot)\right)
$$


Writing

$$
X_{\Gamma, \varepsilon}:=\left\{z \in X ; \min _{\gamma \in \Gamma} \rho_{\gamma}(z)>\varepsilon\right\}
$$

we have the following lemma.

Lemma 6.2. Let $\Gamma$ be a sparse, uniformly separated sequence and let $\varepsilon \leq$ $\sigma(\Gamma)$. The function $v_{r}$ is uniformly bounded on $X_{\Gamma, \varepsilon}$. Moreover, $v_{r}$ satisfies the following estimate: if $\gamma \in \Gamma$ and $\rho_{\gamma}(z)<\sigma$, then

$$
\left|v_{r}(z)-\log \rho_{\gamma}(z)\right| \leq C_{r, \varepsilon}
$$

Proof. Let $z \in X_{\Gamma, \varepsilon}$. Since $\Gamma$ is sparse, there are at most $N=N_{r, 0}$ members of $\Gamma$, say $\gamma_{1}, \ldots, \gamma_{N}$, lying in $D_{r}(z)$, and so

$$
\left|v_{r}(z)\right| \leq \sum_{j=1}^{N}\left(\left|E\left(\gamma_{j}, z\right)\right|+\left|I\left(\gamma_{j}, z\right)\right|\right) \leq \sum_{j=1}^{N}\left(\left|\log \left(\rho_{z}\left(\gamma_{j}\right)\right)\right|+C_{r}\right) .
$$

Note that the number $N$ does not depend on $z$. Since $\varepsilon<\rho_{z}\left(\gamma_{j}\right)<r$, the term involving the logarithm has a bound that depends only on $\varepsilon$ and $r$. We thus see that $v_{r}$ is uniformly bounded on $X_{\Gamma, \varepsilon}$.

Let $\gamma \in \Gamma$. Since $\Gamma$ is sparse, there are at most $N=N_{r, \varepsilon}$ elements of $\Gamma$ that lie in $D_{r}\left(D_{\varepsilon}(\gamma)\right)$. We write $\Gamma \cap D_{r}\left(D_{\varepsilon}(\gamma)\right)=\left\{\gamma_{1}, \ldots, \gamma_{N}\right\}$, where $\gamma_{1}=\gamma$. Again, $N$ does not depend on $z$. Then

$\left|v_{r}(z)-\log \rho_{z}(\gamma)\right| \leq\left(\sum_{j=2}^{N}\left|E\left(\gamma_{j}, z\right)\right|+\sum_{j=1}^{N}\left|I\left(\gamma_{j}, z\right)\right|\right)+\left|E(\gamma, z)-\log \rho_{z}(\gamma)\right|$.

The first sum is bounded because $\sigma(\Gamma)<\rho_{z}\left(\gamma_{j}\right)<r$ for $j=2, \ldots, N$. The second sum is bounded by (6.3), and the third term vanishes. This completes the proof of the lemma.

\section{A function with bumps along $\Gamma$}

Let

$$
d A_{E, \gamma}(\zeta):=d \rho_{\gamma}(\zeta) \wedge * d \rho_{\gamma}(\zeta) \quad \text { and } \quad A_{E, \gamma}(D):=\int_{D} d A_{E, \gamma}
$$

Given a distribution $f$, we consider its regularization

$$
\frac{1}{A_{E, \gamma}\left(D_{\varepsilon}(z)\right)} \int_{D_{\varepsilon}(z)} f d A_{E, \gamma}
$$

using the area element $d A_{E, \gamma}$, where $\gamma \in \Gamma$. 
Observe that

$$
\begin{aligned}
A_{E, \gamma}\left(D_{\varepsilon}(\gamma)\right) & =\int_{D_{\varepsilon}(\gamma)} d \rho_{\gamma} \wedge * d \rho_{\gamma}=\int_{D_{\varepsilon}(\gamma)} \rho_{\gamma} d \rho_{\gamma} \wedge * d E(\gamma, \cdot) \\
& =\int_{0}^{\varepsilon} t\left(\int_{S_{t}(\gamma)} * d E(\gamma, \cdot)\right) d t=2 \pi \int_{0}^{\varepsilon} t d t=\pi \varepsilon^{2}
\end{aligned}
$$

Consider the function

$$
v_{r, \varepsilon}(z)=t \sum_{\gamma \in \Gamma} \frac{1}{\pi \varepsilon^{2}} \int_{D_{\varepsilon}(\gamma)}(E(\zeta, z)-I(\zeta, z)) d A_{E, \gamma}(\zeta)
$$

where $0 \ll t<1$.

Lemma 6.3. The function $v_{r, \varepsilon}$ has the following properties.

(1)

$$
\begin{aligned}
e^{\nu(z)} \Delta v_{r, \varepsilon}(z)=t \sum_{\gamma \in \Gamma} & \frac{1}{2 \varepsilon^{2}} e^{\nu(z)}\left|d \rho_{\gamma}(z)\right|^{2} \mathbf{1}_{D_{\varepsilon}(z)} \\
& -t \sum_{\gamma \in \Gamma} \frac{1}{2 \varepsilon^{2}} \int_{D_{\varepsilon}(\gamma)} \xi_{r}(\cdot, z) d A_{E, \gamma} .
\end{aligned}
$$

In particular,

$$
\lim _{\varepsilon \rightarrow 0} e^{\nu} \Delta v_{r, \varepsilon}=\frac{\pi}{2} t \sum_{\gamma \in \Gamma}\left(e^{\nu} \delta_{\gamma}-\xi_{r}(\gamma, \cdot)\right)
$$

in the sense of distributions.

(2) There exists a positive constant $C_{r, \varepsilon}$ such that

$$
z \in X \Rightarrow-C_{r, \varepsilon} \leq v_{r, \varepsilon}(z) \leq 0
$$

and for any $\gamma \in \Gamma$,

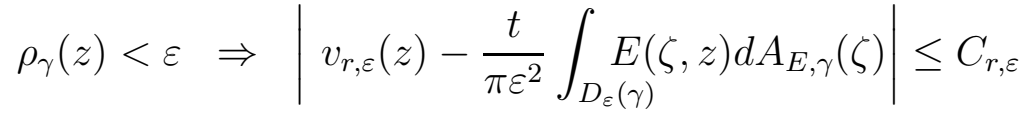

Proof. 1. The formula for the Laplacian is a straightforward calculation, and the limit is a standard consequence of the regularization of currents.

2. Since $E(\zeta, z)=I(\zeta, z)$ whenever $\rho_{z}(\zeta)>r$, we have, in view of formula (6.6),

$$
v_{r, \varepsilon}(z)=\sum_{\gamma \in D_{\varepsilon}\left(D_{r}(z)\right)} \frac{t}{\pi \varepsilon^{2}} \int_{D_{\varepsilon}(\gamma)}(E(\zeta, z)-I(\zeta, z)) d A_{E, \gamma}(\zeta) .
$$


Choose $\gamma \in \Gamma$. Since $\Gamma$ is sparse, there exist $\gamma_{1}, \ldots, \gamma_{N} \in \Gamma-\{\gamma\}$ such that for all $z \in D_{\varepsilon}(\gamma)$

$$
\begin{aligned}
v_{r, \varepsilon}(z)=\frac{t}{\pi \varepsilon^{2}} \int_{D_{\varepsilon}(\gamma)}( & E(\zeta, z)-I(\zeta, z)) d A_{E, \gamma} \\
& +\sum_{j=1}^{N} \frac{t}{\pi \varepsilon^{2}} \int_{D_{\varepsilon}\left(\gamma_{j}\right)}(E(\zeta, z)-I(\zeta, z)) d A_{E, \gamma_{j}}
\end{aligned}
$$

Moreover, $N$ is independent of $\gamma$, and depends only on $r$ and $\varepsilon$. It follows that

$$
\begin{aligned}
& \left|v_{r, \varepsilon}(z)-\frac{t}{\pi \varepsilon^{2}} \int_{D_{\varepsilon}(\gamma)} E(\cdot, z) d A_{E, \gamma}\right| \\
& \quad \leq \frac{t}{\pi \varepsilon^{2}} \int_{D_{\varepsilon}(\gamma)}|I(\cdot, z)| d A_{E, \gamma}+\sum_{j=1}^{N} \frac{t}{\pi \varepsilon^{2}} \int_{D_{\varepsilon}\left(\gamma_{j}\right)}(|E(\cdot, z)|+|I(\cdot, z)|) d A_{E, \gamma_{j}}
\end{aligned}
$$

We have estimates for $I(\zeta, z)$ as in the proof of Lemma 6.2, and since, by uniform separation, $\rho_{z}(\zeta)>\sigma$ for any $\zeta \in D_{\varepsilon}\left(\gamma_{j}\right)$, we can estimate the right hand side by a constant that depends only on $r$. This proves (6.8), and (6.7) follows from (6.8), Lemma 6.2 and the fact that $v_{r} \leq 0$.

Lemma 6.4. For any $z \in D_{\varepsilon}(\gamma)$,

$$
\frac{1}{A_{E, \gamma}\left(D_{\varepsilon}(\gamma)\right)} \int_{D_{\varepsilon}(\gamma)} E(z, \zeta) d A_{E, \gamma}(\zeta) \leq \log \frac{1}{\varepsilon}+\frac{1}{2}
$$

Proof. Observe that if $z \in D_{\varepsilon}(\gamma)$ and $t \in(0, \varepsilon]$, then

$$
\int_{S_{t}(\gamma)} * d_{\zeta} E(z, \zeta)=\int_{D_{t}(\gamma)} d_{\zeta} * d_{\zeta} E(z, \zeta)=2 \pi \mathbf{1}_{D_{t}(\gamma)}(z) \leq 2 \pi .
$$

Applying Green's formula $(2.1)$ with $f=E(z, \cdot)$ and $h=E(\gamma, \cdot)$, we obtain

$$
\int_{S_{t}(\gamma)} E(z, \zeta) * d_{\zeta} E(\gamma, \zeta)=\int_{S_{t}(\gamma)} E(\gamma, \zeta) * d_{\zeta} E(z, \zeta)
$$

We thus have

$$
\begin{aligned}
-\int_{D_{\varepsilon}(\gamma)} \log \rho_{z} d \rho_{\gamma} \wedge * d \rho_{\gamma} & =-\int_{0}^{\varepsilon} t\left(\int_{S_{t}(\gamma)} E(z, \zeta) * d_{\zeta} E(\gamma, \zeta)\right) d t \\
& =-\int_{0}^{\varepsilon} t\left(\int_{S_{t}(\gamma)} E(\gamma, \zeta) * d_{\zeta} E(z, \zeta)\right) d t \\
& =-\int_{0}^{\varepsilon} t \log t\left(\int_{S_{t}(\gamma)} * d_{\zeta} E(z, \zeta)\right) d t \\
& \leq-2 \pi \int_{0}^{\varepsilon} t \log t d t=\pi \varepsilon^{2}\left(\frac{1}{2}-\log \varepsilon\right) .
\end{aligned}
$$

The lemma now follows from (6.6). 


\section{Proof of Theorem 1}

Let $\left(s_{\gamma}\right) \in \mathfrak{B}_{\Gamma}^{2}(\varphi, g)$. We first construct a smooth function $\eta \in L^{2}\left(X, e^{-\varphi} d A_{g}\right)$ that interpolates $\left(s_{\gamma}\right)$. To this end, let $\chi \in \mathcal{C}_{0}^{\infty}([0, \sigma))$ satisfy

$$
0 \leq \chi \leq 1, \quad \chi \mid[0, \sigma / 2] \equiv 1 \quad \text { and } \quad\left|\chi^{\prime}\right| \leq \frac{3}{\sigma}
$$

We define

$$
\eta(z):=\sum_{\gamma \in \Gamma} \chi \circ \rho_{\gamma}(z) s_{\gamma} e^{F_{\gamma}(z)}
$$

where $F_{\gamma}$ is as in Lemma 6.1. Observe that $\eta(\gamma)=s_{\gamma}$ for all $\gamma \in \Gamma$, and that

$$
\begin{aligned}
\int_{X}|\eta|^{2} e^{-\varphi} d A_{g} & =\sum_{\gamma \in \Gamma}\left|s_{\gamma}\right|^{2} \int_{D_{\sigma}(\gamma)}\left|\chi \circ \rho_{\gamma}\right|^{2}\left|e^{2 F_{\gamma}-\varphi}\right| d A_{g} \\
& \leq C \sum_{\gamma \in \Gamma}\left|s_{\gamma}\right|^{2} e^{-\varphi(\gamma)} A_{g}\left(D_{\sigma}(\gamma)\right)<+\infty
\end{aligned}
$$

Next we wish to correct $\eta$ by adding to it a function $U$ that lies in $L^{2}\left(X, e^{-\varphi} d A_{g}\right)$ and vanishes along $\Gamma$. The so-called Hörmander-BombieriSkoda technique is to solve the equation $\bar{\partial} U=\bar{\partial} \eta$ with singular weights, using Theorem 2.6. We will use the singular weight $\tilde{\varphi}:=\varphi+v_{r}$. Lemma 6.2 implies that $\tilde{\varphi}$ is comparable to $\varphi$ on the support of $\bar{\partial} \eta$, which lies in $V_{\sigma}(\gamma):=$ $D_{\sigma}(\gamma)-D_{\frac{\sigma}{2}}(\gamma)$. One computes that

$$
\bar{\partial} \eta=\sum_{\gamma \in \Gamma} \chi^{\prime}\left(\rho_{\gamma}\right) \bar{\partial} \rho_{\gamma} s_{\gamma} e^{F_{\gamma}}
$$

and thus we then have the estimate

$$
\begin{aligned}
\int_{X}|\bar{\partial} \eta|^{2} \tau_{\psi} e^{-\tilde{\varphi}} & \leq \frac{C}{\sigma^{2}} \sum_{\gamma \in \Gamma}\left|s_{\gamma}\right|^{2} e^{-\varphi(\gamma)} \int_{V_{\sigma}(\gamma)}\left|\bar{\partial} \rho_{\gamma}\right|^{2} \tau_{\psi} \\
& \leq \frac{C}{\sigma^{2}} \sum_{\gamma \in \Gamma}\left|s_{\gamma}\right|^{2} e^{-\varphi(\gamma)} \int_{D_{\sigma}(\gamma)} e^{\nu}\left|\bar{\partial} \rho_{\gamma}\right|^{2} e^{-\psi} \\
& \leq C^{\prime} \sum_{\gamma \in \Gamma}\left|s_{\gamma}\right|^{2} e^{-\varphi(\gamma)} \int_{D_{\sigma}(\gamma)} e^{-\psi} \\
& <+\infty
\end{aligned}
$$

where the first inequality follows from Lemma 6.1 and the second to last inequality follows from (4.1). 
Since $D_{f}^{+}(\Gamma)<1$, there exist $r<R_{X}$ and $\delta>0$ such that

$$
\begin{aligned}
e^{\nu}\left(\Delta \tilde{\varphi}+\Theta_{\psi}\right) & =e^{\nu}\left(\Delta \varphi+\Theta_{\psi}\right)+e^{\nu} \Delta v_{r} \\
& \geq e^{\nu}\left(\Delta \varphi+\Theta_{\psi}\right)\left(1-\sum_{\gamma \in \Gamma} \frac{\frac{\pi}{2} \xi_{r}(\cdot, \gamma)}{e^{\nu}\left(\Delta \varphi+\Theta_{\psi}\right)}\right) \\
& >\delta e^{\nu}\left(\Delta \varphi+\Theta_{\psi}\right),
\end{aligned}
$$

where the first inequality follows from (6.4). It follows from the admissibility of the metric $e^{-\psi}|d z|^{2}$ that for $\varepsilon>0$ sufficiently small there exists $\delta>0$ such that

$$
\begin{aligned}
e^{\nu}(\Delta \tilde{\varphi}+\Delta \psi & \left.-\frac{\Delta \tau_{\psi}}{\tau_{\psi}}-\frac{\varepsilon}{\tau_{\psi}^{2}}\left|\frac{\partial \tau_{\psi}}{\partial z}\right|^{2}\right) \\
& =e^{\psi}\left(\tau_{\psi}(\Delta \tilde{\varphi}+\Delta \psi)-\Delta \tau_{\psi}-\frac{\varepsilon}{\tau_{\psi}}\left|\frac{\partial \tau_{\psi}}{\partial z}\right|^{2}\right)>\delta .
\end{aligned}
$$

By Ohsawa's Theorem 2.6, there is a function

$$
U \in L^{2}\left(X, e^{-\tilde{\varphi}} d A_{g}\right) \subset L^{2}\left(X, e^{-\varphi} d A_{g}\right)
$$

such that $\bar{\partial} U=\bar{\partial} \eta$. Moreover, since

$$
e^{-\tilde{\varphi}} \sim \frac{1}{|z-\gamma|^{2}}
$$

for $z$ sufficiently close to $\gamma$, we see that $U(\gamma)=0$ for all $\gamma \in \Gamma$. Thus the function

$$
f:=\eta-U \in \mathscr{B}_{X}^{2}(\varphi, g)
$$

interpolates $\left(s_{\gamma}\right)$, and the proof of Theorem 1 is complete.

\section{Proof of Theorem 2}

Let $\hat{\varphi}:=\varphi+v_{r, \varepsilon}$.

Lemma 8.1. Let $g=e^{-\psi}|d z|^{2}$ be an admissible metric. For each $h \in \mathscr{B}_{X}^{2}(\hat{\varphi}, g)$,

$$
\int_{X}|h|^{2} e^{-\hat{\varphi}} e^{\nu}\left(\Delta \hat{\varphi}+\Theta_{\psi}\right) d A_{g} \geq 0 .
$$

Proof. Consider the function $S=|h|^{2} e^{-(\hat{\varphi}+\psi)}$. Then

$$
\frac{\Delta S}{S}=\Delta \log S+\frac{1}{S^{2}}|\partial S|^{2}=\frac{1}{S^{2}}|\partial S|^{2}+\Delta \log |h|^{2}-\Delta(\hat{\varphi}+\psi)
$$


and thus

$$
e^{\nu} \Delta S \geq-S e^{\nu} \Delta(\hat{\varphi}+\psi)
$$

We claim that

$$
\int_{X} e^{\nu} \Delta S d A_{g}=\int_{X} S \Delta \tau_{\psi}
$$

To prove the claim, let $z_{0} \in X$. Take $\lambda \in \mathcal{C}_{0}^{\infty}([0,1 / 2])$ such that $\lambda(t) \equiv 1$ for $0 \leq t \leq 1 / 4$, and put

$$
\chi_{a}(r):=\lambda\left(r^{2}(1-a)\right) .
$$

Then

$$
\begin{aligned}
\int_{X} e^{-(\psi-\nu)} \Delta S=\int_{X} \tau_{\psi} \Delta S & =\lim _{a \nearrow 1} \int_{X} \tau_{\psi} \chi_{a} \circ \rho_{z_{0}} \Delta S \\
& =\lim _{a \nearrow 1} \int_{X} S \Delta\left(\tau_{\psi} \cdot\left(\chi_{a} \circ \rho_{z_{0}}\right)\right) \\
& =\lim _{a \nearrow 1} \int_{X} S\left(\left(\Delta \tau_{\psi}\right) \chi_{a} \circ \rho_{z_{0}}+\left(\partial \tau_{\psi}\right) \wedge \bar{\partial}\left(\chi_{a} \circ \rho_{z_{0}}\right)\right. \\
& =\lim _{a \nearrow 1} \int_{X} S\left(\left(\Delta \tau_{\psi}\right) \wedge \partial\left(\chi_{a} \circ \rho_{z_{0}}\right)+\tau_{\psi} \circ\left(\chi_{z_{0}} \circ \rho_{z_{0}}\right)\right)
\end{aligned}
$$

where the third equality follows from Stokes' Theorem. Now,

$$
\begin{aligned}
\lim _{a \nearrow 1} \int_{X} S \tau_{\psi} \Delta & \left(\chi_{a} \circ \rho_{z_{0}}\right)=\lim _{a \nearrow 1} \int_{X} S \tau_{\psi}\left(\chi_{a}^{\prime \prime}\left(\rho_{z_{0}}\right)\left|\partial \rho_{z_{0}}\right|^{2}+\chi_{a}^{\prime}\left(\rho_{z_{0}}\right) \Delta \rho_{z_{0}}\right) \\
& =\lim _{a \nearrow 1} \int_{X} S \tau_{\psi}\left(\chi_{a}^{\prime \prime}\left(\rho_{z_{0}}\right)+\frac{\chi_{a}^{\prime}\left(\rho_{z_{0}}\right)}{\rho_{z_{0}}}\right)\left|\partial \rho_{z_{0}}\right|^{2} \\
& =\lim _{a \nearrow 1} \int_{X}|h|^{2} e^{-\hat{\varphi}}\left(\chi_{a}^{\prime \prime}\left(\rho_{z_{0}}\right)+\frac{\chi_{a}^{\prime}\left(\rho_{z_{0}}\right)}{\rho_{z_{0}}}\right) e^{\nu}\left|\partial \rho_{z_{0}}\right|^{2} d A_{g}=0
\end{aligned}
$$

where the last equality follows from (4.1) and the definition of $\chi_{a}$. Thus we have

as claimed. Now,

$$
\int_{X} \tau_{\psi} \Delta S=\int_{X} S \Delta \tau_{\psi}
$$

$$
\int_{X} S \Delta \tau_{\psi}=S e^{\nu}\left(\frac{\Delta \tau_{\psi}}{\tau_{\psi}}\right) d A_{g}
$$

It follows that

$$
\begin{aligned}
\int_{X} S e^{\nu}\left(\Delta \hat{\varphi}+\Theta_{\psi}\right) d A_{g} & =\int_{X} S e^{\nu}\left(\Delta(\hat{\varphi}+\psi)-\frac{\Delta \tau}{\tau}\right) d A_{g} \\
& =\int_{X} S e^{\nu} \Delta(\hat{\varphi}+\psi) d A_{g}-\int_{X} e^{\nu} \Delta S d A_{g} \geq 0
\end{aligned}
$$

The proof is complete. 
Conclusion of the proof of Theorem 2. Let $h \in \mathscr{B}_{X}^{2}(\varphi, g)$. By Lemma 6.3,

$$
\begin{gathered}
e^{\nu(z)}\left(\Delta \hat{\varphi}+\Theta_{\psi}\right)(z)=e^{\nu(z)}\left(\Delta \varphi+\Theta_{\psi}\right)(z)+e^{\nu(z)} \Delta v_{r, \varepsilon}(z) \\
=e^{\nu(z)}\left(\Delta \varphi+\Theta_{\psi}\right)(z)\left(1-t \sum_{\gamma \in \Gamma} \frac{1}{2 \varepsilon^{2}} \int_{D_{\varepsilon}(\gamma)} \frac{\xi_{r}(\zeta, z)}{e^{\nu(z)}\left(\Delta \varphi+\Theta_{\psi}\right)(z)} d A_{E, \gamma}(\zeta)\right. \\
\left.+t \sum_{\gamma \in \Gamma} \frac{1}{\varepsilon^{2}} \frac{e^{\psi(z)}\left|\partial \rho_{\gamma}(z)\right|^{2}}{e^{\nu(z)}\left(\Delta \varphi+\Theta_{\psi}\right)(z)} \mathbf{1}_{D_{\varepsilon}(\gamma)}(z)\right)
\end{gathered}
$$

Applying the hypotheses $D_{f}^{-}(\Gamma)>1$, the admissibility and $g$ and the estimate (4.1), we see therefore that, for $t$ sufficiently close to 1 , there exist $r, \delta, C>0$ such that

$$
e^{\nu}\left(\Delta \hat{\varphi}+\Theta_{\psi}\right) \leq-t e^{\nu}\left(\Delta \varphi+\Theta_{\psi}\right)\left(\delta-C \sum_{\gamma \in \Gamma} e^{2 \psi} \frac{2}{\varepsilon^{2}} \mathbf{1}_{D_{\varepsilon}(\gamma)}\right) .
$$

We then apply Lemma 8.1 to get

$$
\begin{aligned}
\int_{X}|h|^{2} e^{-\varphi} d A_{g} \leq \int_{X}|h|^{2} e^{-\hat{\varphi}} d A_{g} \leq C \int_{X} e^{\nu}\left(\Delta \varphi+\Theta_{\psi}\right)|h|^{2} e^{-\hat{\varphi}} d A_{g} \\
\leq C^{\prime} \sum_{\gamma \in \Gamma} \frac{2}{\varepsilon^{2}} \int_{D_{\varepsilon}(\gamma)} e^{\nu}\left(\Delta \varphi+\Theta_{\psi}\right)|h|^{2} e^{-\hat{\varphi}} d A_{g} \\
\leq C^{\prime \prime} \sum_{\gamma \in \Gamma} \frac{2}{\varepsilon^{2}} \int_{D_{\varepsilon}(\gamma)}|h|^{2} e^{-\hat{\varphi}} d A_{g} \leq C^{\prime \prime \prime} \sum_{\gamma \in \Gamma} \frac{2}{\varepsilon^{2+2 t}} \int_{D_{\varepsilon}(\gamma)}|h|^{2} e^{-\varphi} d A_{g}
\end{aligned}
$$

where the first inequality follows from Lemma 6.3, the third inequality follows from integration of (8.2) together with Lemma 8.1 and the last inequality follows from Lemmas 6.3 and 6.4. Now,

$$
\begin{aligned}
\int_{D_{\varepsilon}(\gamma)} \mid & \left.h\right|^{2} e^{-\varphi} d A_{g}=\int_{D_{\varepsilon}(\gamma)}\left|h e^{-F_{\gamma}}\right|^{2} e^{-\varphi+2 \operatorname{Re} F_{\gamma}} d A_{g} \\
& \leq C e^{-\varphi(\gamma)} \int_{D_{\varepsilon}(\gamma)}\left|h e^{-F_{\gamma}}\right|^{2} d A_{g} \\
& \leq C^{\prime} A_{g}\left(D_{\varepsilon}(\gamma)\right) e^{-\varphi(\gamma)}\left(|h(\gamma)|^{2}+\varepsilon^{2} \sup _{D_{\varepsilon}(\gamma)} \frac{\mid\left(h e^{-F_{\gamma}}\right)^{\prime}}{\left|\partial \rho_{\gamma}\right|^{2}}\right) \\
& \leq C^{\prime} A_{g}\left(D_{\varepsilon}(\gamma)\right) e^{-\varphi(\gamma)}\left(|h(\gamma)|^{2}+\varepsilon^{2} C_{\varepsilon, \sigma} \int_{D_{\sigma}(\gamma)}\left|h e^{-F_{\gamma}}\right|^{2} d A_{g}\right) \\
& \leq C^{\prime} A_{g}\left(D_{\sigma}(\gamma)\right) e^{-\varphi(\gamma)}|h(\gamma)|^{2}+\varepsilon^{2} C^{\prime \prime} A_{g}\left(D_{\varepsilon}(\gamma)\right) \int_{D_{\sigma}(\gamma)}|h|^{2} e^{-\varphi} d A_{g},
\end{aligned}
$$


where the first and last inequalities follow from Lemma 6.1, the second inequality follows from Taylor's theorem, and the third inequality from the Cauchy estimate (4.7).

Next, since $e^{-\psi} \leq C e^{-\nu}$ for some $C>0$, we see by Theorem 4.1 that

$$
A_{g}\left(D_{\varepsilon}(\gamma)\right) \leq C \int_{D_{\varepsilon}(\gamma)} e^{-\nu} \leq C_{o} \int_{D_{\varepsilon}(\gamma)}\left|\partial \rho_{\gamma}\right|^{2}=\pi C_{o} \varepsilon^{2}
$$

for all sufficiently small $\varepsilon$ and some $C$ independent of $\gamma$, where the last equality follows from (6.6). We thus obtain

$$
\begin{array}{r}
\int_{X}|h|^{2} e^{-\varphi} d A_{g} \leq \sum_{\gamma \in \Gamma}\left(\frac{C_{1}}{\varepsilon^{2+2 t}}|h(\gamma)|^{2} e^{-\varphi(\gamma)} A_{g}\left(D_{\sigma}(\gamma)\right)+C_{2} \varepsilon^{2-2 t} \int_{D_{\sigma}(\gamma)}|h|^{2} e^{-\varphi} d A_{g}\right) \\
\leq \sum_{\gamma \in \Gamma}\left(\frac{C_{1}}{\varepsilon^{2+2 t}}|h(\gamma)|^{2} e^{-\varphi(\gamma)} A_{g}\left(D_{\sigma}(\gamma)\right)\right)+C_{2} \varepsilon^{2-2 t} \int_{X}|h|^{2} e^{-\varphi} d A_{g}
\end{array}
$$

By taking $\varepsilon$ sufficiently small, we obtain the left hand side of the sampling inequality in Definition 1.1(2). For the right hand side of the sampling inequality, we argue as follows.

$$
\begin{aligned}
\sum_{\gamma \in \Gamma}|h(\gamma)|^{2} e^{-\varphi(\gamma)} A_{g}\left(D_{\sigma}(\gamma)\right) & =\sum_{\gamma \in \Gamma}\left|h(\gamma) e^{-F_{\gamma}(\gamma)}\right|^{2} e^{-\varphi(\gamma)} A_{g}\left(D_{\sigma}(\gamma)\right) \\
& \leq C \sigma^{2} \sum_{\gamma \in \Gamma} e^{-\varphi(\gamma)} \int_{D_{\sigma}(\gamma)}\left|h e^{-F_{\gamma}}\right|^{2} d A_{g} \\
& \leq C^{\prime} \sum_{\gamma \in \Gamma} \int_{D_{\sigma}(\gamma)}|h|^{2} e^{-\varphi} d A_{g} \leq C^{\prime \prime} \int_{X}|h|^{2} e^{-\varphi} d A_{g}
\end{aligned}
$$

where the first inequality follows from (4.6), the second from Lemma 6.1 and the third from the definition of the separation constant. This proves Theorem 2 .

Acknowledgment. We are grateful to John D'Angelo, Jeff McNeal, Quim Ortega-Cerdà and Eric Schippers for interesting discussions.

\section{References}

[1] Berndtsson, B. and Ortega Cerdì, J.: On interpolation and sampling in Hilbert spaces of analytic functions. J. Reine Angew. Math. 464 (1995), 109-128.

[2] Nakai, M.: On Evans' kernel. Pacific J. Math. 22 (1967), 125-137. 
[3] Nakai, M. And Sario, L.: Classification theory of Riemann Surfaces. Die Grundlehren der mathematischen Wissenschaften, Band 164. SpringerVerlag, New York-Berlin, 1970.

[4] Ohsawa, T.: On the extension of $L^{2}$ holomorphic functions. IV. A new density concept. In Geometry and Analysis on complex manifolds, 157-170. World Sci. Publ., River Edge, NJ, 1994.

[5] Ohsawa, T.: On the extension of $L^{2}$ holomorphic functions. V. Effects of generalization. Nagoya Math. J. 161 (2001), 1-21. (See also Erratum: Nagoya Math. J. 163 (2001), 229.)

[6] Schiffer, M.: The kernel function of an orthonormal system. Duke Math. J. 13 (1946), 529-540.

[7] Schiffer, M. And Spencer, D.: Functionals of finite Riemann surfaces. Princeton University Press, Princeton, N. J., 1954.

[8] SEIP, K.: Density theorems for sampling and interpolation in the Bargmann-Fock space. I. J. Reine Angew. Math. 429 (1992), 91-106.

[9] SeIP, K.: Beurling type density theorems in the unit disk. Invent. Math. 113 (1993), no. 1, 21-39.

[10] Seip, K. And Wallstén, R.: Density theorems for sampling and interpolation in the Bargmann-Fock space. II. J. Reine Angew. Math. 429 (1992), $107-113$.

Recibido: 9 de octubre de 2006

Revisado: 5 de junio de 2007

\author{
Alexander Schuster \\ San Francisco State University \\ San Francisco, CA 94132, USA \\ schuster@sf.su.edu \\ Dror Varolin \\ Stony Brook University \\ Stony Brook, NY 11794, USA \\ dror@math . sunysb . edu
}

The first author is partially supported by NSF grant DMS 0601060. The second author is partially supported by NSF grant DMS0400909. 